\title{
Nanoparticle drug delivery: focusing on the therapeutic cargo
}

“...there is a strong need to focus nanomedicine research on identifying potential therapeutics that will benefit from nanoparticle delivery."

\section{KEYWORDS: abandoned drugs $~$ nanomedicine $"$ nanoparticle drug delivery - reformulation - therapeutics development}

\section{Current efforts in nanoparticle drug delivery}

Nanoparticle (NP) drug delivery has garnered intense academic as well as commercial interest in recent years. Today, there are more than 20 clinically approved NP therapeutics with many more in clinical and preclinical development [1]. Most of the research efforts thus far have focused on the engineering and development of novel NP platforms. By contrast, there has been less attention devoted to studying the types of therapeutic cargo that NPs can deliver and their potential applications. Using the development of NP chemotherapeutics as an example, many of the preclinical and clinical investigations focused on the delivery of four drugs: doxorubicin, daunorubicin, paclitaxel and docetaxel. These drugs account for only two classes of chemotherapeutics, the anthracyclines and the taxanes [2]. Given that NP formulations of all four chemotherapeutics have been translated clinically, it is unlikely that additional research will result in the clinical development of new NP therapeutics containing these drugs. Another rationale to study NP delivery of these therapeutics is to compare a novel NP platform to an existing NP. However, a NP platform that is an excellent delivery vehicle for one drug may be a poor delivery vehicle for another. For example, liposomes are excellent for the delivery of hydrophilic anthracyclines, but efforts to use liposomes to deliver similarly hydrophilic platinum drugs have failed [3]. Therefore, there is a strong need to focus nanomedicine research on identifying potential therapeutics that will benefit from NP delivery.

\section{Taking advantage of the unique biodistribution \& clearance of NPs}

One of the unique properties of NPs is their biodistribution. NPs, as macromolecules, preferentially accumulate in areas of leaky vasculature through the enhanced permeability and retention (EPR) effect. This unique property allows NP therapeutics to target disease processes such as cancer and inflammatory diseases. NPs also possess other properties that are favorable for drug delivery. First, NPs are generally cleared by the monocyte phagocytic system and its cargo is cleared by hepatic clearance. This clearance mechanism is very different than that of most small molecules, which is via the kidneys. Hepatic clearance can be a favorable characteristic as it can potentially lower toxicity. Poor renal function is relatively common among the ill and the elderly. Druginduced nephrotoxicity has been a significant problem that has limited the use of many therapeutics [4]. For example, many cancer patients cannot receive cisplatin therapy owing to poor renal function. In comparison, most patients have normal hepatic function, and hepatotoxicity is much less common than nephrotoxicity [5]. Thus, NP delivery can potentially mitigate a therapeutic's nephrotoxicity and increase its clinical use. Although recent publications have demonstrated the proof-of-principle of this approach in nanomedicine, it has not been fully explored [6]. Another property of NPs is their low distribution in normal organs, such as the heart and lungs. For therapeutics with cardiac or pulmonary toxicities as dose-limiting toxicities, NP delivery of these drugs can lower their toxicity profile. Indeed, the success of liposomal doxorubicin lies in its ability to limit drug distribution to the heart, which in turn lowers cardiac toxicity [7]. Using the same strategy, one can reduce the pulmonary toxicity of therapeutics, such as bleomycin, with NP formulations. The biodistribution of NPs can also lead to novel therapeutic applications. For

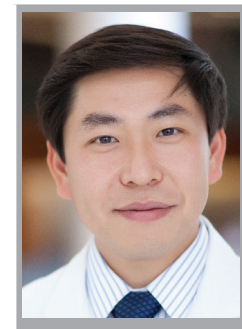

Andrew Z Wang

Department of Radiation Oncology,

Lineberger Comprehensive Cancer

Center, University of North Carolina

School of Medicine, Chapel Hill,

NC 27713, USA

Tel.: +1 9199667700

zawang@med.unc.edu
Future $\because \%$ Medicine part of 
example, preferential accumulation of NPs in tumors and minimal uptake in nearby normal tissue is an ideal property for chemoradiotherapy, a treatment paradigm in oncology [8]. Our group recently showed that NP formulations of docetaxel are more effective than smallmolecule docetaxel in chemoradiotherapy [9].

\section{Taking advantage of the drug delivery properties of NPs}

NPs can be precisely engineered to possess unique size, shape, composition and surface properties. Therefore, NP delivery vehicles allow the modification of some of the most fundamental properties of its therapeutic cargo, such as solubility, immunogenicity and stability. The drug delivery capabilities enabled by NPs opens many new possibilities for therapeutics development. Traditional drug development has relied on chemical modifications to overcome unfavorable properties of lead compounds. While such modifications can be successful, it can also compromise the therapeutic efficacy as well as lead to new toxicities. Thus, the traditional therapeutics development process yields few compounds with both excellent therapeutic efficacy and good pharmacokinetic and toxicity profiles. By contrast, NP therapeutic carriers can address these undesirable properties, such as solubility, stability and toxicity, without the need for chemical modification. With NPs, one can renew the clinical translation potential of many therapeutics that failed clinical development owing to drug delivery challenges. Our group has recently demonstrated the proof of principle of this approach. We evaluated NP delivery of wortmannin (Wtmn). Wtmn is a natural compound that was first discovered more than five decades ago [10]. It was highly promising as a novel cancer therapeutic in preclinical studies, but its clinical translation ultimately failed owing to its poor solubility, low stability (half-life of less than $10 \mathrm{~min}$ ) and high toxicity. While many chemical modifications have been made in attempts to overcome its poor pharmacologic properties over the last several decades, no Wtmn analogues have succeeded in clinical translation. Today, only one Wtmn analogue, PX-866, is still under clinical evaluation. Given that solubility, stability and toxicity can be modified by NP delivery, our group engineered a NP formulation of Wtmn. We demonstrated that NP Wtmn is more soluble, more stable and, most importantly, less toxic than Wtmn [11]. Our work has highlighted one of the great potentials of NP drug delivery: to reformulate abandoned therapeutics.

\section{"The challenge of nanoparticle therapeutic development lies in the regulatory process of clinical translation."}

NPs can also be utilized for the delivery of novel therapeutics with drug delivery challenges. For example, siRNA hold high potential as a novel class of therapeutics. However, their low stability and their need for intracellular delivery prevented their clinical translation. Recent research efforts have focused on utilizing NPs for siRNA delivery. Already, a novel NP formulation of siRNA has been evaluated clinically while many others are in preclinical development $[12,13]$.

\section{Future perspective}

NP-based therapeutics is poised to have significant impact on the treatment of many illnesses. In addition to the strong emphasis on developing effective NP drug delivery platforms, there is a greater need for investigations to expand beyond the commonly studied drugs and to identify new therapeutic cargos as well as novel applications. Compared to clinically approved drugs, there is a far greater number of therapeutics that were once highly promising but ultimately failed clinical development. These therapeutics have well-characterized limitations. NP delivery of these drugs can lead to the development of new classes of therapeutics as well as new therapeutic applications. For example, calicheamicins are some of the most potent antitumor agents, but their clinical development was prevented by their toxicity. NP drug delivery has the potential to lower their toxicity and add these powerful drugs to our arsenal in the fight against cancer.

The challenge of NP therapeutic development lies in the regulatory process of clinical translation. Drug development is high risk and costly. In this process, NP formulations of therapeutics that are already approved for clinical use have an easier regulatory path than that of NP formulations of novel therapeutics. NP reformulations of established drugs have shorter paths to clinical approval, require fewer clinical studies and cost less. On the other hand, NP formulation of novel therapeutics has a higher potential to enable novel therapeutic applications and to improve existing treatment paradigms of diseases. Currently, only liposomes are well established as a NP delivery platform with 
well-known properties. As the field of nanomedicine matures and more clinical studies are conducted with other NP platforms, more NP platforms will become established as proven drug delivery vehicles. These established NP platforms can then be utilized to explore NP delivery of nonapproved therapeutics.

\section{Financial \& competing interests disclosure}

AZ Wang has a consulting agreement with Samyang Biopharmaceuticals, Corporation. He is also the cofounder of Coordination Therapeutics, Inc. AZ Wang is also supported by NIH/National Cancer Institute K12 Career Development Award 5-K12-CA120780-01-05 and UL1TR000083, and NIH Center for Nanotechnology Excellence Grant 1-U54-CA151652-01. The author has no other relevant affiliations or financial involvement with any organization or entity with a financial interest in or financial conflict with the subject matter or materials discussed in the manuscript apart from those disclosed.

No writing assistance was utilized in the production of this manuscript

\section{References}

1 Zhang L, Gu FX, Chan JM, Wang AZ, Langer RS, Farokhzad OC. Nanoparticles in medicine. Therapeutic applications and developments. Clin. Pharmacol. Ther. 83(5), 761-769 (2008).

2 Wang AZ, Langer RS, Farokhzad OC. Nanoparticle delivery of cancer drugs. Annu. Rev. Med. 63, 185-198 (2011)

3 De Jonge MJ, Slingerland M, Loos WJ et al. Early cessation of the clinical development of LiPlaCis, a liposomal cisplatin formulation. Eur. J. Cancer 46(16), 3016-3021 (2010).

4 Naughton CA. Drug-induced nephrotoxicity. Am. Fam. Physician 78(6), 743-750 (2008).

5 King PD, Perry MC. Hepatotoxicity of chemotherapy. Oncologist 6(2), 162-176 (2001).

6 Sengupta P, Basu S, Soni S et al. Cholesteroltethered platinum II-based supramolecular nanoparticle increases antitumor efficacy and reduces nephrotoxicity. Proc. Natl Acad. Sci. USA 109(28), 11294-11299 (2012).

7 O'Brien ME, Wigler N, Inbar M et al. Reduced cardiotoxicity and comparable efficacy in a Phase III trial of pegylated liposomal doxorubicin $\mathrm{HCl}$ (CAELYX/Doxil) versus conventional doxorubicin for first-line treatment of metastatic breast cancer. Ann. Oncol. 15(3), 440-449 (2004).

\section{Werner ME, Foote MB, Wang AZ.} Chemoradiotherapy of human tumors: novel approaches from nanomedicine. Curr. Pharm. Des. 18(19), 2830-2837 (2012).

9 Werner ME, Copp JA, Karve S et al. Folate-targeted polymeric nanoparticle formulation of docetaxel is an effective molecularly targeted radiosensitizer with efficacy dependent on the timing of radiotherapy. ACS Nano 5(11), 8990-8998 (2011).
10 Workman P, Clarke PA, Raynaud FI, Van Montfort RL. Drugging the PI3 kinome: from chemical tools to drugs in the clinic. Cancer Res. 70(6), 2146-2157 (2010).

11 Karve S, Werner ME, Sukumar R et al. Revival of the abandoned therapeutic wortmannin by nanoparticle drug delivery. Proc. Natl Acad. Sci. USA 109(21), 8230-8235 (2012).

12 Davis ME, Zuckerman JE, Choi $\mathrm{CH}$ et al. Evidence of RNAi in humans from systemically administered siRNA via targeted nanoparticles. Nature 464(7291), 1067-1070 (2010).

13 Leuschner F, Dutta P, Gorbatov R et al. Therapeutic siRNA silencing in inflammatory monocytes in mice. Nat. Biotech. 29(11), 1005-1010 (2011). 